LA-UR-01-3562

Approved for public release;

distribution is unlimited.

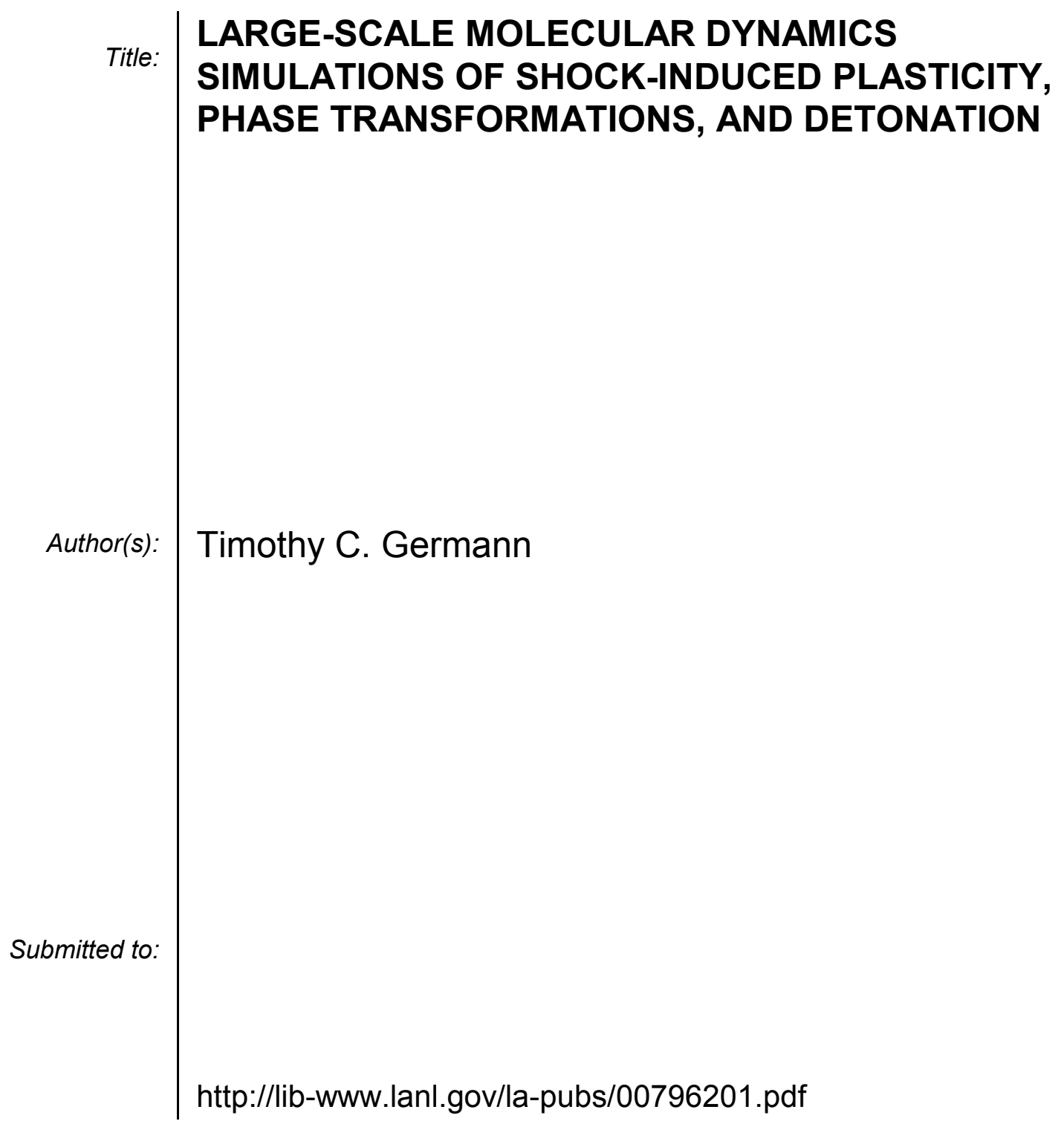

Los Alamos National Laboratory, an affirmative action/equal opportunity employer, is operated by the University of California for the U.S. Department of Energy under contract W-7405-ENG-36. By acceptance of this article, the publisher recognizes that the U.S. Government retains a nonexclusive, royaltyfree license to publish or reproduce the published form of this contribution, or to allow others to do so, for U.S. Government purposes. Los Alamos National Laboratory requests that the publisher identify this article as work performed under the auspices of the U.S. Department of Energy. Los Alamos National Laboratory strongly supports academic freedom and a researcher's right to publish; as an institution, however, the Laboratory does not endorse the viewpoint of a publication or guarantee its technical correctness. 


\title{
LARGE-SCALE MOLECULAR DYNAMICS SIMULATIONS OF SHOCK-INDUCED PLASTICITY, PHASE TRANSFORMATIONS, AND DETONATION
}

\author{
Timothy C. Germann* \\ Applied Physics Division (X-7), Los Alamos National Laboratory, Los Alamos, NM 87545
}

\begin{abstract}
Modern computers enable routine multimillion-atom molecular dynamics simulations of shock propagation in solids using realistic interatomic potentials, and offer a direct insight into the atomistic processes underlying plasticity, phase transformations, and the detonation of energetic materials. Past, present, and prospects for future simulations will be discussed in the context of prototypical systems for each of these three classes of problems. Initial samples ranging from perfect single crystals, to those with specific isolated defects, to full-fledged polycrystalline materials will be considered.
\end{abstract}

\section{INTRODUCTION}

Atomistic simulation methods, particularly nonequilibrium molecular dynamics (MD), offer a great and largely untapped potential for the investigation of shockwave processes in solids [1]. Only recently has it been conclusively demonstrated that largescale MD simulations can give steady plastic (or split elastic-plastic) waves with a rich nanostructure [2] that may be directly compared with ultrafast X-ray diffraction measurements [3]. Shockinduced phase transformations (either solid-solid or solid-melt), multiple shocks (including ramp wave loading), unloading processes such as rarefaction shocks, spallation, and ejecta, and shock-induced chemistry are just a few of the phenomena for which MD simulations over the coming decade should be able to provide a great deal of insight.

This paper will briefly review some of the recent achievements using classical molecular dynamics, and those which may reasonably be expected in the near future. The use of quantum molecular dynamics techniques, including density-functional and tight-binding methods, to study shock-compressed

\footnotetext{
* Work done in collaboration with Brad Lee Holian, Kai Kadau, and Peter S. Lomdahl (LANL); Niels G. Jensen (UC Davis/LBL); Jean-Bernard Maillet (CEA); and Ramon Ravelo (UT El Paso/LANL).
}

materials [4], is another promising field, but will not be considered further.

\section{PROTOTYPICAL SYSTEMS}

Three prototypical systems will be discussed here, representative of three classes of shockinduced behavior in solids, namely (1) plasticity, (2) polymorphic phase transformations, and (3) detonation of energetic materials.

The majority of molecular dynamics simulations for solids have focused on close-packed metals, represented either by pair potentials such as LennardJones 6-12, or embedded atom method (EAM) potentials [5], which add an embedding term dependent on the local electron density to a pair potential. Holian and coworkers [6] first demonstrated that steady shock waves in three-dimensional solids could be modeled using MD, with a transition from a purely elastic response to plastic flow when the Hugoniot pressure jump is roughly equal to the shear modulus. These early (ca. 1980) simulations, with up to $10^{4}$ atoms, exhibited slippage (i.e., stacking faults) along one, or at most two, $\{111\}$ planes for shocks traveling in the $\langle 100\rangle$ direction. With the great advances in computer power and in parallel molecular dynamics algorithms [7], Holian and Lomdahl [2] demonstrated that 3-D simula- 
tions with $10^{7}$ atoms are feasible on moderate-sized (12-CPU) multiprocessor computers. These studies confirmed the results of earlier simulations (e.g., regarding the linear $u_{s}$ vs. $u_{p}$ Hugoniot, the threshold for plasticity, ...), with patterns of intersecting stacking faults being generated on all four available $\{111\}$ planes. Shortly thereafter, Zhakhovskiı̌ et al. [8] showed that smooth fine-grained shock profiles could also be obtained using a time-averaging technique with much smaller samples than the spatial averaging over large cross-sectional samples. Further simulations have investigated the crystallographic orientation dependence in both the elasticplastic [9] and melting [10] regimes.

Recently, simulations have been carried out using various EAM potentials for iron, to investigate the $\alpha \rightarrow \epsilon$ phase transformation under shock loading [11]. It is rather remarkable how fast the timescale for this diffusionless process is; for overdriven shocks in perfect single crystals, the bcc $\rightarrow$ hcp transformation is basically complete within a few lattice planes, although subsequent grain annealing is observed over longer timescales. Since the published potentials were all fit to zero-pressure data (or at best, an approximate Rose "universal equation of state" for the bcc phase), it is not surprising that details of the experimental Hugoniot are not quantitatively reproduced, especially above the transformation threshold. But the fact that qualitative aspects such as the bcc-hcp orientation relationships, transformation kinetics, etc., for different shock directions appears to be independent of the actual potential leads us to believe that these represent the true response of shock-compressed perfect single-crystal iron.

The third and final class of behavior concerns shock-induced chemical reactions, specifically detonation. Reactive empirical bond order (REBO) potentials developed by Brenner, White, and coworkers $[12,13]$ in the early 1990 s exhibit many of the properties of condensed phase explosives, including a critical flyer plate velocity for initiation [13], detonation velocity independent of initiation conditions [13], delayed (homogeneous) initiation for low velocity impact [14], and a critical width for detonation of 2-D ribbons [15]. All of these studies (as well as the present work) are for the original $2 \mathrm{AB}$ $\rightarrow \mathrm{A}_{2}+\mathrm{B}_{2}$ model exothermic reaction, but shock simulations using REBO potentials for ozone [16] and hydrocarbons [17] have also been carried out.

In the remainder of this paper, we will discuss past, present, and future MD simulations for each of these three systems with three types of initial samples: perfect single crystals, single crystals with isolated defects, and polycrystalline materials.

\section{PERFECT SINGLE CRYSTALS}

Single-crystal simulations are beginning to provide quantitative predictions about behavior which can be measured at the macroscale. Holian and Lomdahl [2] demonstrated that with sample crosssections as large as $100 \times 100$ unit cells, quantitative measurements of the stacking fault density could be made and shown to closely follow the total volumetric strain $u_{p} / u_{s}$ across the shock front. Furthermore, analysis of the smooth stress profiles obtained by either time- or (in this case) space-averaging shows that the strain rate dependence on the pressure rise $P$ is $\dot{\varepsilon} \simeq P^{3.3}$, in remarkably good agreement with experimental measurements for metals.

Strachan and coworkers [18] have studied the spallation of perfect crystals of bcc Ta and fcc Ni using EAM potentials. Although the sample sizes were relatively small (10 to 20 thousand atoms), they were able to extract void volume distributions at different times which exhibit a power-law behavior $N(V) \propto V^{-\tau}$ over several orders of magnitude. The critical exponent $\tau \sim 2.2$, which corresponds to that for 3-D percolation. Similar studies using larger samples with preexisting defects such as voids, inclusions, or grain boundaries would be of great interest since spall failure at lesser strain rates (closer to most experiments) could be realized.

With improved potential models, phase transformation simulations may reach a similar level of predictive capacity as well. However, MD simulations of detonation will likely be more valuable in a strictly model capacity, since typical reaction zones ( $\mu \mathrm{m}$ to $\mathrm{mm}$ ) of actual explosives are well beyond the capability of MD simulations for at least another decade. Simulations using REBO models subjected to flyer plate $[13,14]$ or supported piston $[12,16]$ impact have been carried out in 2D and 3D; here we focus on our 2D supported piston results. The measured Hugoniot is shown in Fig. 1. At very slow piston velocities (below about $300 \mathrm{~m} / \mathrm{s}$ ), a steady 


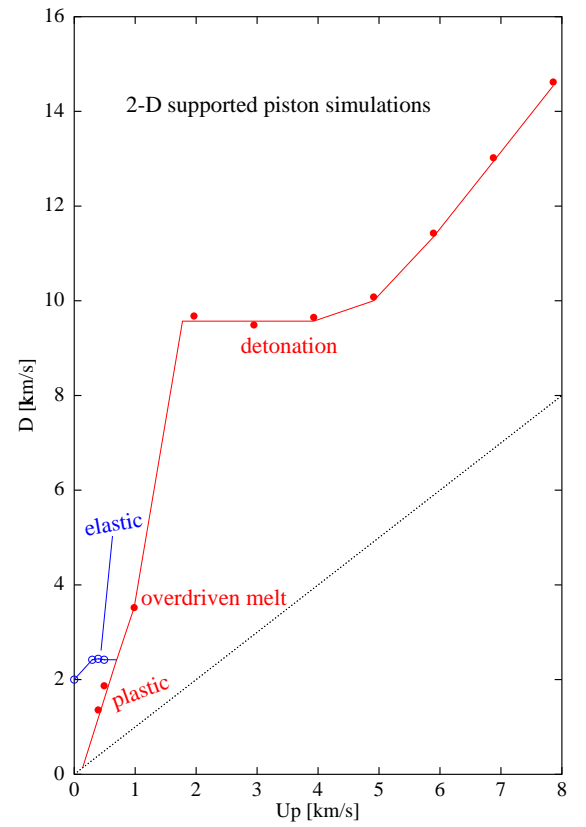

FIGURE 1: Measured Hugoniot (shock velocity vs. particle velocity) for the 2-D AB crystal.

elastic wave is maintained, but soon thereafter one finds split elastic-plastic structure (Fig. 2) followed by a fluid region due to the extremely low melting point of the $\mathrm{AB}$ model. For piston velocities greater than $\sim 1.6 \mathrm{~km} / \mathrm{s}$, a steady-state detonation is initiated. Velocities just above this threshold clearly indicate that initiation takes place by homogeneneous nucleation far behind an intial compressive wave, which is subsequently overtaken by the detonation wave. The detonation velocity is nearly constant, $\simeq 9.5 \mathrm{~km} / \mathrm{s}$, until for $u_{p}>4.5 \mathrm{~km} / \mathrm{s}$ an overdriven detonation wave exists.

\section{STUDIES OF ISOLATED DEFECTS}

Our simulations indicate that point defects such as vacancies are insufficiently strong stress concentrators to initiate plastic deformation [2] or phase transformations below the perfect single-crystal threshold. Similarly, replacing an AB molecule in the molecular solid by an A- radical leads to a few localized reactions for $u_{p}<1.6 \mathrm{~km} / \mathrm{s}$, but does not seem to lower the detonation threshold appreciably (a few percent at most).

However, more extended defects or grain boundaries may readily act as heterogeneous nucleation

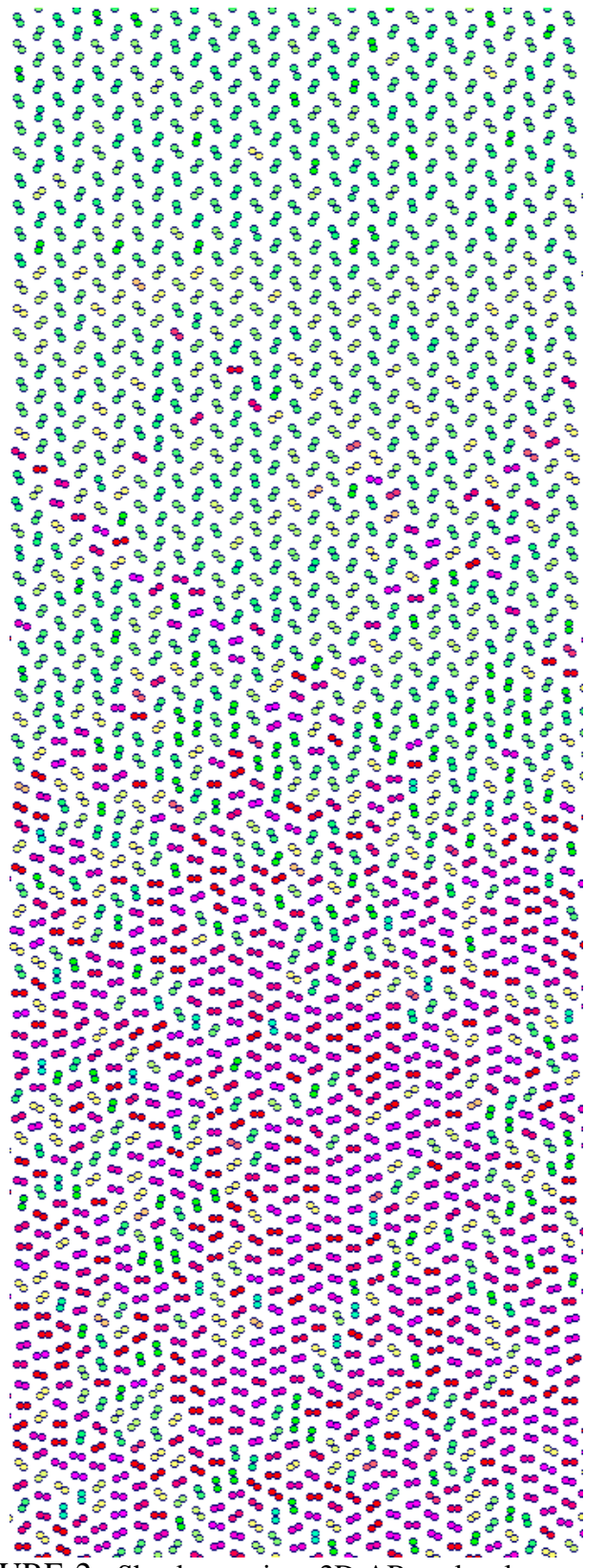

FIGURE 2: Shockwave in a $2 \mathrm{D}$ AB molecular crystal with $u_{p}=0.49 \mathrm{~km} / \mathrm{s}$ (i.e., below the detonation threshold), with molecules colored by their orientation. The unshocked herringbone lattice is at the top, and at the bottom is the shocked state corresponding to a (highly defective) $90^{\circ}$ rotation of the original lattice. An intermediate elastic precursor consists of diagonal lines of single molecules which have rotated by varying amounts, but return to the original configuration upon release. 


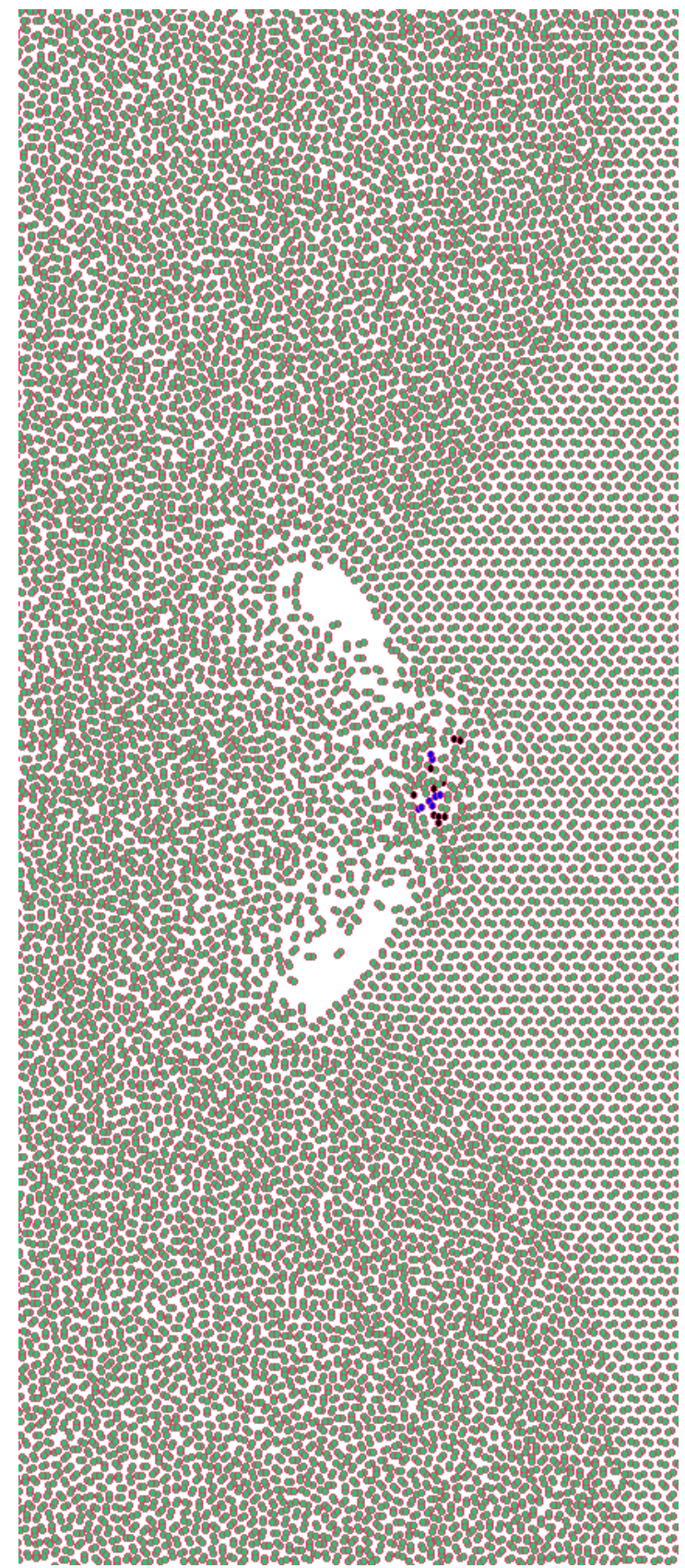

FIGURE 3: Heterogeneous nucleation of detonation at a $10 \mathrm{~nm}$ radius circular void, $6 \mathrm{ps}$ after a nonreactive shock wave reaches the left side of the void. At later times, a steady planar detonation wave is obtained. Atoms are colored according to their bonds, with blue for products and black for multiply bonded atoms.

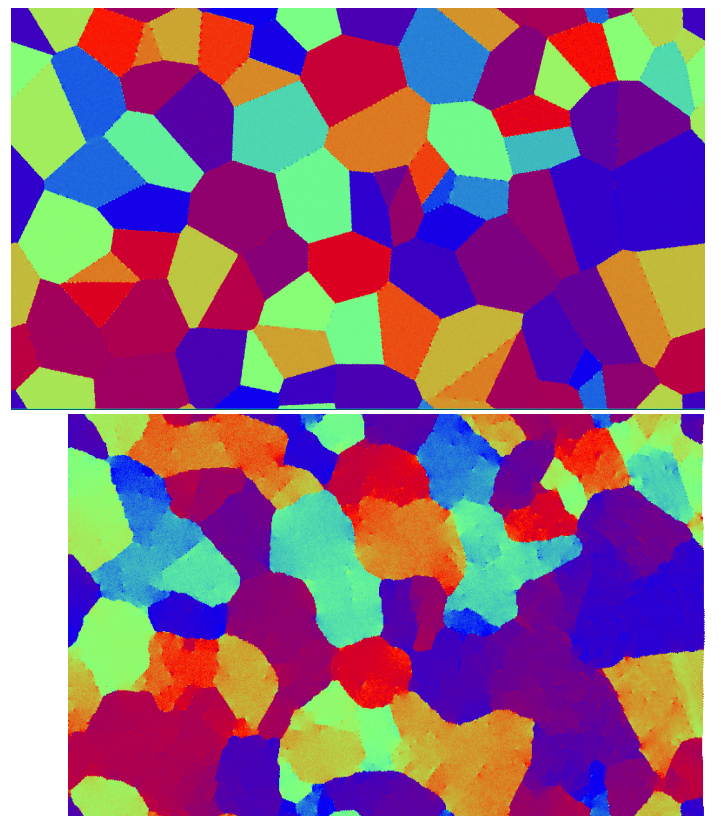

FIGURE 4: Polycrystalline 2-D Lennard-Jones sample ( 2 million atoms) before (top) and after (bottom) shock compression from the left side. Atoms are colored according the the local orientation of the hexagonal lattice.

sites, as demonstrated by using a warped piston to initiate plastic flow in fcc Lennard-Jonesium [2] well below the perfect-crystal threshold. Simulations of bec iron with a missing half-plane of atoms (which can relax into a parallel pair of edge dislocation lines) also show that phase transformation nucleation may be induced below the usual threshold.

One important question which can be addressed using such simulations is what role various types of defects (voids, inclusions, dislocations, ...) play in "hot spot" initiation of detonation. For instance, we have found that large voids in a $2 \mathrm{D} \mathrm{AB}$ crystal can substantially lower the perfect-crystal detonation threshold, from $1.6 \mathrm{~km} / \mathrm{s}$ to around $1.1 \mathrm{~km} / \mathrm{s}$ for $10 \mathrm{~nm}$ radius circular voids. An example of this process is shown in Fig. 3 for $u_{p}=1.38 \mathrm{~km} / \mathrm{s}$. One can clearly see initiation in this case occuring due to the impact of a converging jet of atoms ejected from the opposide sides of the voids, and not by collision of the two waves propagating around the void.

\section{POLYCRYSTALLINE MATERIALS}

We have recently begun carrying out some pre- 

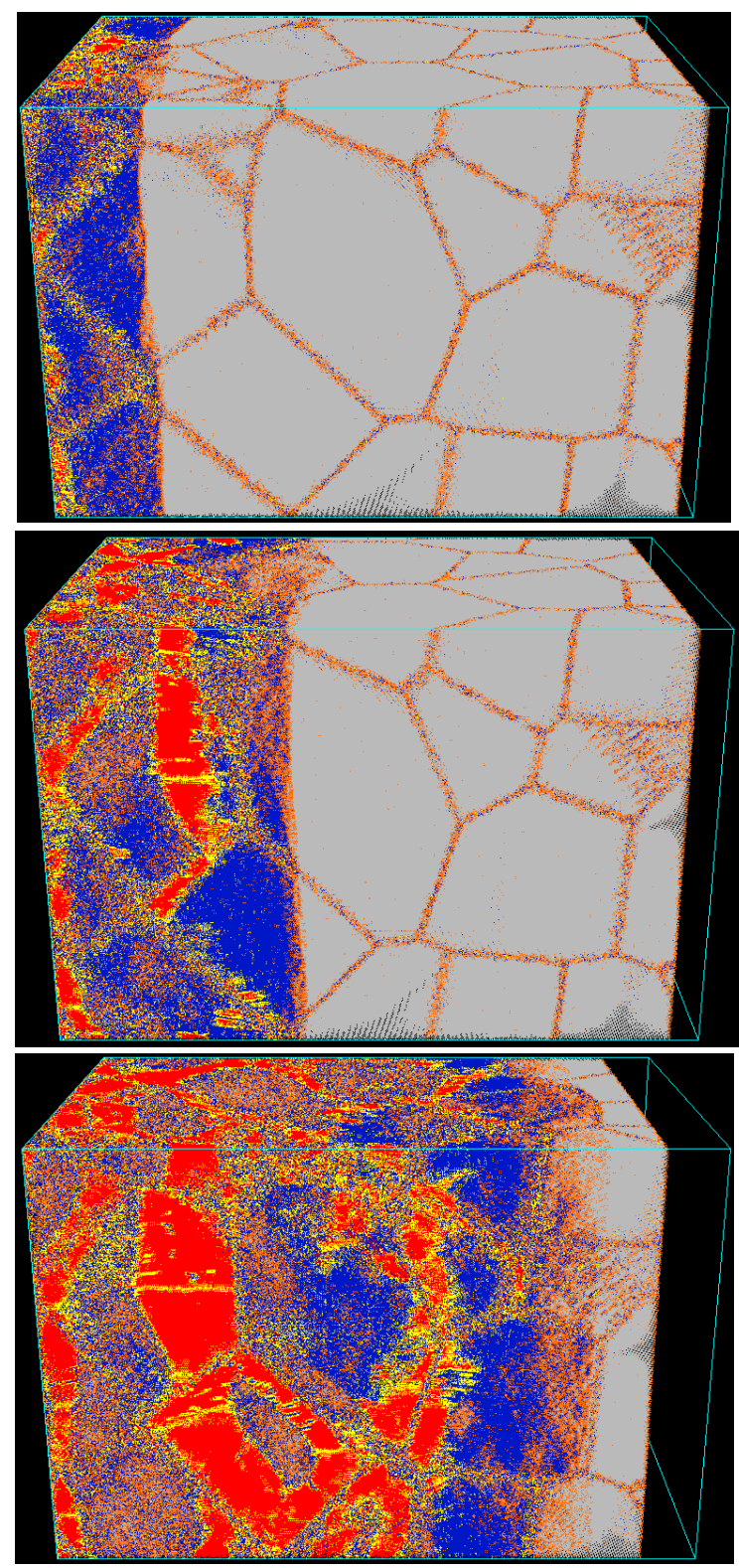

FIGURE 5: Polycrystalline iron sample at $2.2 \mathrm{ps}, 4.5$ ps, and 9.5 ps after impact with a piston at the left (not shown). The sample contains 32 grains and a total of 24 million atoms; the size before shock compression was 58 $\mathrm{nm} \times 58 \mathrm{~nm} \times 87 \mathrm{~nm}$. Atoms are color-coded by the number of neighbors $n$ within $2.75 \AA$ : the unshocked bcc sample $(n=8)$ is grey, uniaxially compressed bcc $(n=$ $10)$ is blue, the transformed close-packed region $(n=12)$ is red, and other values (colors) primarily correspond to grain and twin boundaries. liminary simulations of nanocrystalline materials. The difficulties here are twofold: (1) sample preparation that generates a realistic distribution of grain sizes, orientations (texturing), and relative orientations (grain boundary energies); and (2) samples sufficiently large that many grains are averaged over in the transverse directions, and that a steady-state shock wave is achieved in the longitudinal direction.

A two-dimensional example is shown in Fig. 4. The sample is generated with a Voronoi construction: we randomly select 80 grain center positions and orientations, and fill each grain with a triangular lattice of the given orientation until the midpoint between two grain centers is reached. This initial state is then annealed for $O\left(10^{3}\right)$ timesteps (corresponding to a few ps) to at least partially relax the grain boundaries, before being subjected to shock loading. In this case the piston velocity is below the perfect-crystal Hugoniot elastic limit (at least for the various triangular lattice orientations which we have studied), but animations clearly show dislocations propagating from the grain boundaries through each crystallite. As seen in the bottom panel of Fig. 4, this process greatly distorts the previously linear grain boundaries, and leaves behind a significant dislocation density in several of the grains as well. The shock wave is considerably broadened due to the distribution of shock velocities in each grain and the scattering off of grain boundaries, so even for this 2 million-atom system, a steady-state shock profile is not yet attained.

Such simulations may also be carried out in 3-D, but simulation sizes of $\gg 10^{7}$ atoms are necessary to have a reasonable number of grains and sufficiently small surface-to-volume ratio of each grain. One preliminary example for iron is shown in Fig. 5, where a 32-grain initial configuration was generated in a manner similar to that for the previous example, and then driven at $u_{p}=725 \mathrm{~m} / \mathrm{s}$ towards a momentum mirror [2] at the left. For this particular EAM potential, this piston velocity is about $15 \%$ below the perfect-crystal transformation threshold for shocks in the $\langle 001\rangle$ direction [11]. Again, we see nucleation of the bcc $\rightarrow$ hcp transformation at grain boundaries, which then slowly proceeds inwards for each grain. As before, a steady shock profile is not yet attained, but in this case the number of grains in each transverse cross-section is too small to ever 
expect steady-state behavior.

\section{CONCLUSIONS}

The prospects are very good for using MD simulations on perfect or nearly perfect crystals to systematically study shock phenomena, particularly the effects of various heterogeneities, in a quantitative capacity for metal plasticity and phase transformation processes. On the other hand, energetic material and polycrystal studies are more likely to remain in a qualitative, model-building capacity for the forseeable future. Even $10^{9}$ atoms are not enough to represent a micron-scale cube of material, so direct MD simulations of realistic polycrystalline samples with grain sizes on the order of $10 \mu \mathrm{m}$ are impractical, and unnecessary. Instead a more beneficial approach would involve using MD simulations to calibrate the governing interactions for models (such as the discrete element method) used in mesomechanical studies of shock compression [19], thus providing a link between interatomic potentials on the sub-nanometer scale and mesoscopic behavior on the sub-millimeter scale.

\section{ACKNOWLEDGEMENTS}

Fruitful discussions with Mark Elert, Jerry Erpenbeck, Jim Hammerberg, Ed Kober, John Mintmire, Alejandro Strachan, Carter White, and Sergey Zybin are gratefully acknowledged. This work was carried out under the auspices of the U.S. Dept. of Energy at Los Alamos National Laboratory under Contract W-7405-ENG-36.

\section{REFERENCES}

[1] Holian, B.L., Germann, T.C., Lomdahl, P.S., Hammerberg, J.E., and Ravelo, R., in Shock Compression of Condensed Matter-1999, edited by M.D. Furnish et al., AIP Conference Proceedings 505, New York, 2000, pp. 35-41.

[2] Holian, B.L., and Lomdahl, P.S., Science 280, 2085-2088 (1998)

[3] Loveridge-Smith, A., et al., Phys. Rev. Lett. 86, 2349-2352 (2001)

[4] For instance, see Kress, J.D., Bickham, S.R., Collins, L.A., Holian, B.L., and Goedecker, S., Phys. Rev. Lett. 83, 3896-3899 (1999).

[5] Daw, M.S., Foiles, S.M., and Baskes, M.I., Mater. Sci. Rep. 9, 251-310 (1993).
[6] Holian, B.L., Phys. Rev. A 37, 2562-2568 (1988).

[7] The SPaSM ("Scalable Parallel Short-range Molecular dynamics") code is described in: Lomdahl, P.S, Tamayo, P., Grønbech-Jensen, N., and Beazley, D.M., in Proceedings of Supercomputing 93, edited by G.S. Ansell, IEEE Computer Society Press, Los Alamitos, CA, 1993, pp. 520527; Beazley, D.M., and Lomdahl, P.S., Computers in Physics 11(3), 230-238 (1997); see also http://bifrost.lanl.gov/MD/MD.html.

[8] Zhakovskiǔ, V.V., Zybin, S.V., Nishihara, K., and Anisimov, S.I., Phys. Rev. Lett. 83, 1175-1178 (1999).

[9] Germann, T.C., Holian, B.L., Lomdahl, P.S., and Ravelo, R., Phys. Rev. Lett. 84, 5351-5354 (2000).

[10] Zhakovskiǔ, V.V., Zybin, S.V., Nishihara, K., and Anisimov, S.I., Prog. Theor. Phys. Supp. 138, 223 228 (2000).

[11] Kadau, K., Germann, T.C., Lomdahl, P.S., and Holian, B.L., these proceedings.

[12] Robertson, D.H., Brenner, D.W., and White, C.T., Phys. Rev. Lett. 67, 3132-3135 (1991).

[13] Brenner, D.W., Robertson, D.H., Elert, M.L., and White, C.T., Phys. Rev. Lett. 70, 2174-2177 (1993); 76, 2202(E) (1996).

[14] Robertson, D.H., Brenner, D.W., and White, C.T., Mat. Res. Soc. Symp. Proc. 296, 183-186 (1993).

[15] White, C.T., Robertson, D.H., Swanson, D.R., and Elert, M.L., in Shock Compression of Condensed Matter-1999, edited by M.D. Furnish et al., AIP Conference Proceedings 505, New York, 2000, pp. 377-380.

[16] Barrett, J.J.C., Robertson, D.H., Elert, M.L., and White, C.T., in Shock Compression of Condensed Matter-1997, edited by S.C. Schmidt et al., AIP Conference Proceedings 429, New York, 1998, pp. 329-331.

[17] Stuart, S.J., Tutein, A.B., and Harrison, J.A., $J$. Chem. Phys. 112, 6472-6486 (2000); Elert, M.L., Swanson, D.R., and White, C.T., in Shock Compression of Condensed Matter-1999, edited by M.D. Furnish et al., AIP Conference Proceedings 505, New York, 2000, pp. 283-286.

[18] Strachan, A., Cağin, T., and Goddard, W.A., Phys. Rev. B 63, 060103(R) (2001).

[19] For instance, see Balokhonov, R.R., Makarov, P.V., Romanova, V.A., and Smolin, I.Y., Comput. Mater. Sci. 16, 355-361 (1999); Yano, K., and Horie, Y., Phys. Rev. B 59, 13672-13680 (1999). 